\title{
Algebraic Analysis of Bifurcation and Limit Cycles for Biological Systems *
}

\author{
Wei Niu ${ }^{1}$ and Dongming Wang ${ }^{2,1}$ \\ ${ }^{1}$ Laboratoire d'Informatique de Paris 6, Université Pierre et Marie Curie - CNRS, \\ 104 avenue du Président Kennedy, F-75016 Paris, France \\ ${ }^{2}$ LMIB - SKLSDE - School of Science, Beihang University, Beijing 100083, China \\ wei.niu@etu.upmc.fr, Dongming.Wang@lip6.fr
}

\begin{abstract}
In this paper, we show how to analyze bifurcation and limit cycles for biological systems by using an algebraic approach based on triangular decomposition, Gröbner bases, discriminant varieties, real solution classification, and quantifier elimination by partial CAD. The analysis of bifurcation and limit cycles for a concrete two-dimensional system, the self-assembling micelle system with chemical sinks, is presented in detail. It is proved that this system may have a focus of order 3, from which three limit cycles can be constructed by small perturbation. The applicability of our approach is further illustrated by the construction of limit cycles for a two-dimensional Kolmogorov prey-predator system and a three-dimensional Lotka-Volterra system.
\end{abstract}

\section{Introduction}

Many physical and biological phenomena may be modeled mathematically by dynamical systems. For most nonlinear dynamical systems, it is difficult to find their exact analytical solutions (if such solutions exist at all). Therefore, to understand the phenomenon described by a complex dynamical system, it is necessary to study its behaviors such as stability, bifurcation, and limit cycles qualitatively. The qualitative analysis of such behaviors is a highly nontrivial task and for biological systems it is often performed experimentally by means of numerical simulation and visualization (see, e.g., [2]). Dynamical systems usually involve parameters and their behaviors may change dramatically as the parameters vary. Symbolic and algebraic computation provides powerful tools for rigorous qualitative analysis of dynamical systems with parameters.

We are concerned with biological phenomena that may be modeled by autonomous systems of ordinary differential equations of the form

$$
\frac{\mathrm{d} x_{1}}{\mathrm{~d} t}=p_{1}\left(\boldsymbol{\lambda}, x_{1}, \ldots, x_{n}\right), \ldots, \frac{\mathrm{d} x_{n}}{\mathrm{~d} t}=p_{n}\left(\boldsymbol{\lambda}, x_{1}, \ldots, x_{n}\right),
$$

where $p_{1}, \ldots, p_{n}$ are polynomials (or rational functions) in $\boldsymbol{\lambda}$ and $x_{1}, \ldots, x_{n}$ with real coefficients and $\boldsymbol{\lambda}=\lambda_{1}, \ldots, \lambda_{m}$ are real parameters independent of the derivation variable $t$. As usual, each $x_{i}$ is a function of $t$.

\footnotetext{
* This work has been supported by the Chinese National Key Basic Research (973) Projects 2004CB318000 and 2005CB321901/2 and the SKLSDE Project 07-003.
} 
For biological systems of the form (1.1) with $p_{1}, \ldots, p_{n}$ being rational functions, it has been demonstrated in $[15,16,23,24]$ how their steady states can be detected and how the stability of the steady states can be analyzed by using symbolic and algebraic computation. The technique of linearization used for stability analysis there may fail at bifurcation points because near such points the behavior of system (1.1) may differ qualitatively from that of its linearized system and bifurcation may occur (see $[10,14,29]$ ). Even for autonomous systems there may be many different bifurcating situations, whose study requires sophisticated mathematical methods and effective computational tools. Among them there is one important situation, called Hopf bifurcation (or AndronovHopf bifurcation). In this situation, the Jacobian matrix of system (1.1) has a pair of purely imaginary eigenvalues but no other eigenvalue with zero real part. Hopf bifurcation leads to the birth of limit cycles from a steady state of the dynamical system, when the steady state changes its stability via the movements of the imaginary eigenvalues away from the imaginary axis.

The behavior of limit cycles (i.e., isolated periodic orbits) has been observed in many physical and biological systems. The study of limit cycles is a subject of active research and of great interest in pure mathematics for more than a century. Determining the existence and the exact number of limit cycles is a difficult problem even for planar autonomous polynomial differential systems. This problem is closely related to the longstanding 16 th problem of D. Hilbert $[8,18,20,28$, 29]. The analysis of bifurcation and limit cycles is not only a challenging problem in the qualitative theory of dynamical systems, but also of practical value now for our understanding of the qualitative behaviors of biological systems.

In this paper, we analyze bifurcation and construct limit cycles for a concrete two-dimensional (planar) system, the self-assembling micelle system with chemical sinks [2], by using an algebraic approach based on triangular decomposition [21,25], Gröbner bases [3,9], discriminant varieties [11], real solution classification [27], and quantifier elimination by partial CAD [6]. The stability and bifurcation for this biological system have been analyzed initially in [16], where our general algebraic approach is described in generality. Here we show that this system may have a focus of order 3 , from which three limit cycles can be constructed by small perturbation. The applicability of our approach is further illustrated by the construction of limit cycles for a Kolmogorov prey-predator system and a Lotka-Volterra system. The paper is structured as follows. In the following section, we explain how bifurcation and limit cycles for planar autonomous systems may be analyzed by using algebraic methods. The analysis of bifurcation and the construction of limit cycles for the self-assembling micelle system with chemical sinks are presented in detail in Section 3. Section 4 contains the results of analysis of bifurcation and limit cycles for the two-dimensional cubic Kolmogorov prey-predator system and the competitive three-dimensional Lotka-Volterra system. The paper concludes with a few remarks in Section 5.

Prior to the algebraic analysis of bifurcation investigated by the authors [16], other relevant work has been done by El Kahoui and Weber [7] who applied quantifier elimination [6] to Hopf bifurcation analysis, by $\mathrm{Lu}$ and others in [12, 
13] where limit cycles for a class of biological systems are analyzed by using similar algebraic approaches, and by other researchers in the purely mathematical context of bifurcation and limit cycles (see, e.g., $[8,18,19])$. Hopf bifurcation analyzed in the mathematical context is usually for systems in the canonical form with the origin as their steady state. The biological systems with parameters that we need to analyze are not given in such a form. Transformation of the systems to the canonical form results in complicated expressions and the introduction of new variables and thus increases the computational complexity for the subsequent algebraic analysis considerably.

\section{Bifurcation Analysis for Two-dimensional Systems}

In this section, we consider the case of Hopf bifurcation for $n=2$ in which limit cycles may bifurcate. In this case, the characteristic polynomial of the Jacobian matrix of system (1.1) at a steady state has a pair of purely imaginary roots and the differential system is known as of center-focus type.

Let $n=2$, the variables $x_{1}$ and $x_{2}$ be renamed $x$ and $y,(\bar{x}, \bar{y})$ be a steady state of system (1.1), and $\overline{\boldsymbol{J}}$ be the Jacobian matrix

$$
\left[\begin{array}{l}
a(\boldsymbol{\lambda}, x, y) b(\boldsymbol{\lambda}, x, y) \\
c(\boldsymbol{\lambda}, x, y) d(\boldsymbol{\lambda}, x, y)
\end{array}\right]
$$

of $(1.1)$ at $(\bar{x}, \bar{y})$. Then the characteristic polynomial of $\overline{\boldsymbol{J}}$ has a pair of purely imaginary roots only if $(\bar{x}, \bar{y})$ satisfies the conditions

$$
a+d=0, \quad-a^{2}-b c>0 .
$$

The problems of deciding whether the steady states of system (1.1) without parameters satisfy the conditions (2.1) and determining the conditions on the parameters $\boldsymbol{\lambda}$ (when they are present) for the steady states of (1.1) to satisfy (2.1) may be solved by using algebraic methods based on quantifier elimination [6], real solution classification [27], and discriminant varieties [11].

Now assume that the conditions (2.1) are satisfied. We want to analyze the bifurcation and limit cycles for system (1.1) with $n=2$. In the literature there are several methods for studying this problem [8], e.g., by using Poincaré-Birkhott normal forms, Liapunov constants, succession functions, averaging, and intrinsic harmonic balancing. To deal with the problem, we make a linear transformation

$$
x=-\frac{1}{c} Y-\frac{a}{c \delta} X+\bar{x}, \quad y=-\frac{1}{\delta} X+\bar{y}, \quad t=\frac{\tau}{\delta},
$$

where $\delta=\sqrt{-a^{2}-b c}$. Then system (1.1) with $n=2$ is transformed into the following canonical form

$$
\frac{\mathrm{d} X}{\mathrm{~d} \tau}=Y+P(\boldsymbol{\lambda}, \delta, \bar{x}, \bar{y}, X, Y), \quad \frac{\mathrm{d} Y}{\mathrm{~d} \tau}=-X+Q(\boldsymbol{\lambda}, \delta, \bar{x}, \bar{y}, X, Y),
$$

where $P$ and $Q$ are polynomials in $\mathbb{Q}(\boldsymbol{\lambda}, \delta)[\bar{x}, \bar{y}, X, Y]$ and $\mathbb{Q}$ denotes the field of rational numbers. 
According to Liapunov's theorem [14,29], if one can construct an analytic function $L(X, Y) \in \mathbb{Q}(\boldsymbol{\lambda}, \delta)[\bar{x}, \bar{y}, X, Y]$, called a Liapunov function, such that $L(X, Y)$ is positive in the neighborhood of a steady state and the differential of $L(X, Y)$ along the integral curve of the differential system is definite, then the stability of the steady state can be determined by the sign of the differential. In [20], Wang described a procedure based on the classical method of J. H. Poincaré for constructing such a function for (2.3). Using this procedure, one can compute so-called Liapunov constants (or focal values) $v_{3}, v_{5}, \ldots, v_{2 j+1}, \ldots$ in $\boldsymbol{\lambda}, \delta, \bar{x}, \bar{y}$ such that the differential $L(X, Y)$ along the integral curve of (2.3) is of the form

$$
\frac{\mathrm{d} L(X, Y)}{\mathrm{d} \tau}=v_{3} Y^{4}+v_{5} Y^{6}+\cdots+v_{2 j+1} Y^{2 j+2}+\cdots .
$$

Then the stability of the steady state $(0,0)$ for system $(2.3)$ and thus $(\bar{x}, \bar{y})$ for system (1.1) is determined by the sign of $\mathrm{d} L / \mathrm{d} \tau$ and therefore by the sign of the first nonzero Liapunov constant $v_{2 k+1}$. Namely, we have the following simple criteria.

Theorem 1 ([20, 29]). For any given parametric values $\overline{\boldsymbol{\lambda}}$ of $\boldsymbol{\lambda}$ and steady state $(\bar{x}, \bar{y})=\left(\bar{x}_{1}, \bar{x}_{2}\right)$ of system (1.1) with $n=2$,

(a) if there is an integer $k \geq 1$ such that $v_{3}=\cdots=v_{2 k-1}=0$ but $v_{2 k+1} \neq 0$, then $(\bar{x}, \bar{y})$ is unstable when $v_{2 k+1}>0$, and asymptotically stable when $v_{2 k+1}<0$;

(b) if $v_{2 j+1}=0$ for all $j=1,2, \ldots$, then $(\bar{x}, \bar{y})$ is stable of center type, but not asymptotically stable.

In case (a), the steady state $(\bar{x}, \bar{y})$ of system (1.1) is said to be a focus of order $k$. In case (b), $(\bar{x}, \bar{y})$ is said to be a center of (1.1). By Theorem 1 (a), the problem of determining the stability of a focus is reduced to that of determining the signs of the Liapunov constants and thus may be solved by using the algebraic methods mentioned above.

It is possible to construct limit cycles in the neighborhood of a focus (so-called small-amplitude limit cycles) by perturbation. According to the fundamental theorem on bifurcation and limit cycles [1, p. 239], if the steady state $(0,0)$ of (2.3) is a focus of order $k$, then by perturbing (2.3), $k$ small-amplitude limit cycles near $(0,0)$ may bifurcate. To construct such limit cycles, one needs to find the real solutions of $v_{3}=\cdots=v_{2 k-1}=0, v_{2 k+1} \neq 0$. This may be done by a combination of the methods and software tools of triangular decomposition, Gröbner bases, and real solving. When a real solution is found, one may perturb the system so that the Liapunov constants of the perturbed system have alternate signs, i.e., $v_{i} v_{i+2}<0$ for $i=3, \ldots, 2 k+1$ in the neighborhood of $(0,0)$. Then $k$ (and at most $k$ ) small-amplitude limit cycles may be created from the focus.

Determining necessary and sufficient conditions for $(0,0)$ to be a center from the computed Liapunov constants is a tougher issue because the conditions in Theorem 1 (b) are given by infinitely many equalities (in a finite number of variables). It may be tackled by using algebraic computation (as shown in the extensive literature on the derivation of center conditions, see, e.g., [22, Sect. 10.3]) 
together with some sophisticated mathematical techniques. The main difficulty in deriving center conditions and searching for differential systems having foci of high order from Liapunov constants comes from the large polynomials that cannot be effectively managed even on a powerful computer.

\section{Bifurcation and Limit Cycles for Self-assembling Micelle Systems with Chemical Sinks}

Consider the following dissipative dynamical system studied in $[2,16]$ :

$$
\frac{\mathrm{d} x}{\mathrm{~d} t}=\mu-x y^{2}-x(r+\alpha)=p, \quad \frac{\mathrm{d} y}{\mathrm{~d} t}=r x+x y^{2}-\eta y=q .
$$

The rate coefficients $\alpha$ and $\eta$ represent combined quantities that include a common flow-rate component as well as separate chemical sink-rates for each species, and $\mu$ and $r$ are intrinsic parameters.

We want to derive conditions for system (3.1) to be of center-focus type and for its foci to be stable and to construct small-amplitude limit cycles by means of bifurcation analysis.

\subsection{Bifurcation Analysis}

To determine the conditions under which system (3.1) is of center-focus, we first compute the purely lexicographical (plex) Gröbner basis of $\{p, q\}$ with $y \prec x$ : the basis consists of two polynomials

$$
g_{1}=\eta y^{3}-\mu y^{2}+r \eta y+\alpha \eta y-r \mu, \quad g_{2}=\alpha x+\eta y-\mu .
$$

The system $g_{1}=0, g_{2}=0$ has real solutions for any parametric values of $\mu, r$, and $\alpha \neq 0, \eta \neq 0$. Therefore, for $\alpha \eta \neq 0$ system (3.1) always has steady states. Let $\alpha \eta \neq 0$ and $\bar{y}=w$ be a real root of $g_{1}$. Then $\bar{x}=(\mu-\eta w) / \alpha$ is a real root of $g_{2}$. The Jacobian matrix of $(3.1)$ at $(\bar{x}, \bar{y})$ is

$$
\left[\begin{array}{ll}
a & b \\
c & d
\end{array}\right]=\left[\begin{array}{cc}
-\left(w^{2}+r+\alpha\right) & -\frac{2 w(\mu-\eta w)}{\alpha} \\
r+w^{2} & \frac{2 w(\mu-\eta w)}{\alpha}-\eta
\end{array}\right] .
$$

System (3.1) becomes of center-focus type when

$$
\begin{aligned}
& f_{1}=\alpha w^{2}+2 \eta w^{2}-2 \mu w+r \alpha+\alpha \eta+\alpha^{2}=-(a+d) \alpha=0, \\
& f=(\alpha-\eta) w^{2}-r \eta+r \alpha+\alpha^{2}=a^{2}+b c+(a+d)\left(r+w^{2}\right)<0 .
\end{aligned}
$$

Note that

$$
f_{2}=\left.g_{1}\right|_{y=w}=\eta w^{3}-\mu w^{2}+r \eta w+\alpha \eta w-r \mu=0 .
$$

From $f_{1}=f_{2}=0, f<0$ and by using the Maple package DISCOVERER (developed by B. Xia, see http://www.is.pku.edu.cn/ ${ }^{\sim}$ xbc/software.html) or DV (developed by G. Moroz and F. Rouillier, see http://fgbrs.lip6.fr/Software/DV/), one can obtain conditions $(\mathrm{CF})$ in the parameters $\eta, \mu, r, \alpha$ for (3.1) to be of center-focus type, under which limit cycles may bifurcate from $(\bar{x}, \bar{y})$. The con- 
ditions $(\mathrm{CF})$ are quite complicated and we do not produce them here. It may also be proved easily (e.g., by using DISCOVERER) that, if $\eta=\alpha$, then there are no real values of $\mu, r, \alpha \neq 0$ that satisfy $(\mathrm{CF})$. This confirms the conclusion in [2] that Hopf bifurcations are absent and (CF) hold only for non-physical values of $\alpha$ in this case. However, there do exist real values of $\mu, r, \eta, \alpha$ such that $0 \neq \eta \neq \alpha \neq 0$ and (CF) hold, as we will see clearly below.

Under the conditions ( $\mathrm{CF})$, system (3.1) may be transformed by (2.2) into the following canonical form

$$
\frac{\mathrm{d} X}{\mathrm{~d} \tau}=Y+\frac{\delta}{\alpha} Q, \quad \frac{\mathrm{d} Y}{\mathrm{~d} \tau}=-X+Q,
$$

corresponding to $(2.3)$, where $\delta=\sqrt{-f}$ and

$$
\begin{aligned}
& Q=\frac{\gamma}{\left(r+w^{2}\right) \delta^{3}} X^{2}-\frac{2 \alpha w}{\left(r+w^{2}\right) \delta^{2}} X Y-\frac{\alpha\left(r+\alpha+w^{2}\right)}{\left(r+w^{2}\right) \delta^{4}} X^{3}+\frac{\alpha}{\left(r+w^{2}\right) \delta^{3}} X^{2} Y, \\
& \gamma=2 \alpha w^{3}+\eta w^{3}-\mu w^{2}+2 \alpha^{2} w+2 r \alpha w+r \eta w-r \mu .
\end{aligned}
$$

The Liapunov constants of (3.2) may be computed by the function miscel [licon] from the Epsilon library [22] (see http://www-calfor.lip6.fr/ ${ }^{\sim}$ wang/epsilon). The first Liapunov constant is

$$
\begin{aligned}
v_{3}= & \frac{\alpha}{3\left(r+w^{2}\right) \delta^{3}}-\frac{r+\alpha+w^{2}}{\left(r+w^{2}\right) \delta^{3}}-\frac{2 \alpha w \gamma}{3\left(r+w^{2}\right)^{2} \delta^{5}}+\frac{2 w \gamma}{3 \alpha\left(r+w^{2}\right)^{2} \delta^{3}} \\
& +\frac{2 \gamma^{2}}{3 \alpha\left(r+w^{2}\right)^{2} \delta^{5}}
\end{aligned}
$$

whose numerator $\bar{v}_{3}$, when expanded, has 34 terms. The numerators $\bar{v}_{5}, \ldots, \bar{v}_{13}$ of the subsequent 5 Liapunov constants $v_{5}, \ldots, v_{13}$ consist of $384,1969,6616,17504$, 39467 terms and are of total degrees $18,28,38,48,58$ in the variables $\eta, r, \alpha, w, \delta, \mu$, respectively.

Let us first take $\eta=1$ as in [2]. We want to determine real values of $r, \alpha$ and $\mu$ such that $a+d=0, a^{2}+b c<0, v_{3}=v_{5}=0$ but $v_{7} \neq 0$, so that the steady state $(0,0)$ of $(3.2)$ is a focus of order 3 . For this purpose, we first compute the plex Gröbner basis $\mathcal{G}$ of $\left\{f_{1}, f_{2}, \delta^{2}+f, \bar{v}_{3}, \bar{v}_{5}\right\}$ under the variable order $r \prec \alpha \prec w \prec \delta \prec \mu$ using the Groebner package in Maple. It is found that the first polynomial in $\mathcal{G}$ may be factorized as

$$
\alpha r^{7}(4 r+1)^{2}(24 r-1)^{2}\left(16 r^{2}-24 r+1\right) h,
$$

where

$$
\begin{aligned}
h= & 1474560 r^{7}-3997696 r^{6}+4549632 r^{5}-4503808 r^{4} \\
& -4966528 r^{3}-928256 r^{2}-24396 r+2025 .
\end{aligned}
$$

Since all the physical parameters are required to be positive, we can assume that $\alpha \delta \neq 0$ and $r>0$, so the factors $\alpha, r, 4 r+1$ in (3.4) need not be considered. Let $z$ be a new indeterminate and fix the order for the variables as $r \prec \alpha \prec$ $w \prec \delta \prec \mu \prec z$. The reduced plex Gröbner bases of both $\mathcal{G} \cup\{24 r-1, z \alpha \delta-1\}$ and $\mathcal{G} \cup\left\{16 r^{2}-24 r+1, z \alpha \delta-1\right\}$ are equal to $\{1\}$, so the factors $24 r-1$ and 
$16 r^{2}-24 r+1$ need not be considered either. The technique used here to exclude the case $\alpha \delta=0$ by introducing $z$ and adding the equation $z \alpha \delta-1$ is standard and known as Rabinowich's trick.

Now compute the plex Gröbner basis $\mathcal{G}^{*}$ of $\mathcal{G} \cup\{h, z \alpha \delta-1\}$ with respect to the above variable order: $\mathcal{G}^{*}$ consists of 6 polynomials, of which the first is $h$. The set of zeros of $\mathcal{G}^{*}$ covers all the zeros $(\bar{r}, \bar{\alpha}, \bar{\mu}, \bar{w}, \bar{\delta})$ of $\mathcal{G}$ with $\bar{r}>0$ and $\bar{\alpha} \bar{\delta} \neq 0$. Isolating the real zeros of $\mathcal{G}^{*}$ using the Maple package RS (developed by F. Rouillier, see http://fgbrs.lip6.fr/ $/$ roullier/Software/RS/), we find that $\mathcal{G}^{*}$ has 8 real zeros. Among these zeros there is one and only one $(\bar{r}, \bar{\alpha}, \bar{\mu}, \bar{w}, \bar{\delta})$ for which $\bar{r}, \bar{\alpha}, \bar{\mu}, \bar{w}, \bar{\delta}$ are all positive. For this positive zero, we have

$$
\begin{aligned}
& \bar{r} \approx 0.033247029312587, \quad \bar{\alpha} \approx 0.347417369934422, \\
& \bar{\mu} \approx 1.165669793409291, \quad \bar{w} \approx 0.702121202169318 .
\end{aligned}
$$

It may be easily verified by using RS that the real zero $(\bar{r}, \bar{\alpha}, \bar{\mu}, \bar{w})$ satisfies $f<0$, and that for $\eta=1$ and $(r, \alpha, \mu)=(\bar{r}, \bar{\alpha}, \bar{\mu}), v_{3}=0, v_{5}=0$ and $v_{7}<0$. Therefore, the steady state

$$
(\bar{x}, \bar{y})=\left(\frac{\bar{\mu}-\bar{w}}{\bar{\alpha}}, \bar{w}\right) \approx(1.334270049098212,0.702121202169318)
$$

is an asymptotically stable focus of order 3 and thus three limit cycles may bifurcate from $(\bar{x}, \bar{y})$ for system (3.1) with small perturbation.

Theorem 2. For $\eta=1$, there is one and only one set of positive values $\bar{r}, \bar{\alpha}, \bar{\mu}$ as shown in (3.5) for the parameters $r, \alpha, \mu$ such that system (3.1) has a focus of order 3 . This focus is located at $(\bar{x}, \bar{y})=((\bar{\mu}-\bar{w}) / \bar{\alpha}, \bar{w})$ and is asymptotically stable, where $\bar{w}$ is as in (3.5).

In the next subsection, we will show how to construct a perturbed polynomial differential system of (3.2) that has three small-amplitude limit cycles near $(0,0)$.

\subsection{Construction of Limit Cycles}

To construct small-amplitude limit cycles, let us consider the following perturbed system of (3.2):

$$
\frac{\mathrm{d} X}{\mathrm{~d} \tau}=\lambda X+Y+\frac{\delta}{\alpha} Q_{1}, \quad \frac{\mathrm{d} Y}{\mathrm{~d} \tau}=-X+\lambda Y+Q_{2},
$$

where

$$
\begin{aligned}
Q_{1}= & \frac{\gamma}{\left(r+w^{2}\right) \delta^{3}} X^{2}-\frac{2 \alpha w}{\left(r+w^{2}\right) \delta^{2}} X Y-\frac{\alpha\left(r+\alpha+w^{2}\right)}{\left(r+w^{2}\right) \delta^{4}} X^{3} \\
& +\left[\frac{\alpha}{\left(r+w^{2}\right) \delta^{3}}+\omega\right] X^{2} Y, \\
Q_{2}= & \frac{\gamma}{\left(r+w^{2}\right) \delta^{3}} X^{2}-\frac{2 \alpha w}{\left(r+w^{2}\right) \delta^{2}} X Y-\left[\frac{\alpha\left(r+\alpha+w^{2}\right)}{\left(r+w^{2}\right) \delta^{4}}+\xi\right] X^{3} \\
& +\left[\frac{\alpha}{\left(r+w^{2}\right) \delta^{3}}+\theta\right] X^{2} Y,
\end{aligned}
$$


and $\gamma$ is as in (3.3). The first three Liapunov constants of (3.7) computed by the Epsilon function miscel [licon] are as follows:

$$
\left.v_{3}\right|_{\lambda=0}=\frac{\theta}{3}+\frac{\Gamma_{1}}{3 \alpha \delta^{5}\left(r+w^{2}\right)^{2}},
$$

where $\Gamma_{1}$ consists of 34 terms and is of total degree 8 in the variables $r, \alpha, w, \delta, \mu$;

$$
\left.v_{5}\right|_{\lambda=0, \theta=0}=\frac{\Gamma_{2} \omega+\Gamma_{3} \xi+\Gamma_{4}}{45 \alpha^{3} \delta^{11}\left(r+w^{2}\right)^{4}},
$$

where $\Gamma_{2}, \Gamma_{3}, \Gamma_{4}$ consist of $62,62,384$ terms and are of total degrees $15,15,12$ in $r, \alpha, w, \delta, \mu$, respectively;

$$
\left.v_{7}\right|_{\lambda=0, \theta=0, \omega=0}=\frac{\Gamma_{5} \xi^{2}+\Gamma_{6} \xi+\Gamma_{7}}{1890 \alpha^{5} \delta^{17}\left(r+w^{2}\right)^{6}},
$$

where $\Gamma_{5}, \Gamma_{6}, \Gamma_{7}$ consist of $90,581,1969$ terms and are of total degrees 25,22, 19 in $r, \alpha, w, \delta, \mu$, respectively.

Obviously, if $\lambda=\theta=0$, then $v_{3}=0$, so $\Gamma_{1}=0$. Thus we may use $\Gamma_{1}$ to reduce the numerator $\bar{v}_{5}$ of $\left.v_{5}\right|_{\lambda=0, \theta=0}$ to obtain a remainder $R_{5}$ (which consists of 80 terms and is of total degree 20 in $r, \alpha, w, \delta, \mu, \omega, \xi)$ such that $\bar{v}_{5}=U_{5} \Gamma_{1}+R_{5}$ for some $U_{5}$. Similarly, we can use $\Gamma_{1}$ to reduce the numerator of $\left.v_{7}\right|_{\lambda=0, \theta=0, \omega=0}$ to get a remainder $R_{7}$, which consists of 348 terms and is of total degree 20 in $r, \alpha, w, \delta, \mu, \xi$.

Note that $r>0, \alpha>0, \delta>0, w>0, \mu>0$ and $\Gamma_{1}$ vanishes at any zero of $\mathcal{G}^{*}$. So one can choose $-1 \ll \theta<0$, such that $v_{3}<0$. Next, we determine the condition on $\omega$ and $\xi$ such that $\mathcal{G}^{*}=0$ and $R_{5}>0$. Using the DV package, we compute a minimal discriminant variety $\Delta_{1}(\omega, \xi)$ of $\mathcal{G}^{*}=0, R_{5} \neq 0$. By means of partial CAD, we can obtain 18 cells of the two-dimensional real space $\mathbb{R}^{2}$ of $\omega, \xi$ decomposed by $\Delta_{1}=0$. Choosing one sample point in each cell and computing the sign of $R_{5}$ at each sample point, we find that under the assumption $r>0$, $\alpha>0, \delta>0, w>0, \mu>0,|\xi|<1$, if $\omega<3 / 32$, then $R_{5}>0$. Finally, we determine the condition on $\xi$ such that $\mathcal{G}^{*}=0$ and $R_{7}<0$. Using the DV package, we compute a minimal discriminant variety $\Delta_{2}(\xi)$ of $\mathcal{G}^{*}=0, R_{7} \neq 0$ : $\Delta_{2}$ has 10 real zeros $\xi_{1}<\cdots<\xi_{10}$, which divide the real space $\mathbb{R}$ of $\xi$ into 11 intervals. We choose one sample point in each interval, compute the sign of $R_{7}$ at each sample point, and thus find that, under the assumption $r>0, \alpha>0$, $\delta>0, w>0, \mu>0$, if $\xi_{3}<\xi<\xi_{10}$, then $R_{7}<0$, where

$$
\xi_{3} \in\left[-\frac{2845043675}{4294967296},-\frac{22760349399}{34359738368}\right], \quad \xi_{10} \in\left[\frac{2379513075}{67108864}, \frac{19036104601}{536870912}\right] .
$$

Therefore, if we choose sufficiently small values for the perturbation variables successively such that

$$
0<\lambda \ll-\theta \ll|\omega| \ll|\xi| \ll 3 / 32,
$$

then the Liapunov constants of (3.7) have alternate signs, i.e.,

$$
v_{1}=\lambda>0, \quad v_{3}<0, \quad v_{5}>0, \quad v_{7}<0 .
$$

In this case, the stability of the focus $(\bar{x}, \bar{y})$ of $(3.7)$ turns over three times 
and thus three limit cycles in a small neighborhood of $(\bar{x}, \bar{y})$ can bifurcate. The creation of small-amplitude limit cycles by choosing sufficiently small values for the perturbation variables to change the stability of a focus is a typical technique in bifurcation theory (see [18], [28, pp. 214-215], and [29, pp. 272-273]).

Theorem 3. Three limit cycles may bifurcate in the neighborhood of $(\bar{x}, \bar{y})$ for system (3.1) with $(\eta, r, \alpha, \mu)=(1, \bar{r}, \bar{\alpha}, \bar{\mu})$ and small perturbation, where $\bar{x}, \bar{y}, \bar{r}$, $\bar{\alpha}, \bar{\mu}$ are as in (3.6) and (3.5). The perturbed differential system (3.7) of (3.2) remains polynomial and the perturbation variables $\lambda, \theta, \omega, \xi$ may take sufficiently small values satisfying (3.8).

\subsection{Conditions for the Existence of Foci}

Condition for (3.1) to have a focus of order 3. It has been shown in Section 3.1 that if $\eta=1$, then system (3.1) may have a focus of order 3. Now we want to derive the condition on $\eta$ for system (3.1) to be of center-focus type and have a (positive) focus of order 3 . This can be done by deriving the condition on $\eta$ such that $a+d=0, a^{2}+b c<0, v_{3}=v_{5}=0, v_{7} \neq 0, r>0, \alpha>0$, $\mu>0, w>0$. To do so, we compute a minimal discriminant variety $V$ of $f_{1}=0$, $f_{2}=0, f+\delta^{2}=0, \bar{v}_{3}=0, \bar{v}_{5}=0, \bar{v}_{7} \neq 0, r \neq 0, \alpha \neq 0, \mu \neq 0, w \neq 0, \delta \neq 0$ (where $\bar{v}_{i}$ is the numerator of $v_{i}$ for $i=3,5,7$ ) using DV and check the signs of the polynomials at the sample points of the cells of $\mathbb{R}$ decomposed by $V$. It is then found that for any positive value of $\eta$, the semi-algebraic system $a+d=0$, $a^{2}+b c<0, v_{3}=0, v_{5}=0, v_{7} \neq 0, r>0, \alpha>0, \mu>0, w>0$ has at least one real solution for $(r, \alpha, \mu, w)$.

Theorem 4. For any positive value of $\eta$, there exists at least one set of positive values for the parameters $r, \alpha, \mu$ such that system (3.1) is of center-focus type and has a positive focus of order 3 .

Absence of focus of order 4 for (3.1). Finally, we show that system (3.1) does not have any focus of order greater than 3 and thus one cannot construct 4 small-amplitude limit cycles from a focus by perturbation. For this end, we do not take any values for the parameters in (3.1). We want to decide whether there exist real values of $r, \alpha, \mu, \eta$ such that $a+d=0, a^{2}+b c<0$, and $v_{3}=v_{5}=v_{7}=0$, so that the steady state $(0,0)$ of $(3.2)$ is a center or a focus of order greater than 3 .

Assume that $\alpha \delta \eta r \neq 0$. We compute the plex Gröbner basis $\mathcal{G}$ of $\left\{f_{1}, f_{2}, \delta^{2}+\right.$ $\left.f, \bar{v}_{3}, \bar{v}_{5}, \bar{v}_{7}, z \alpha \delta \eta r-1\right\}$ under the variable order $r \prec \alpha \prec w \prec \delta \prec \mu \prec \eta \prec$ $z$ using FGb, an efficient Gröbner basis package developed by Faugère (see http://fgbrs.lip6.fr/jcf/Software/FGb): the basis is $\{1\}$. This implies that the system $a+d=0, a^{2}+b c<0, v_{3}=0, v_{5}=0, v_{7}=0, r \neq 0, \alpha \neq 0, \eta \neq 0$ has no solution for $r, \alpha, \mu, \eta$, so the following result is proved.

Theorem 5. There are no nonzero values for the parameters $r, \alpha, \mu, \eta$ such that system (3.1) is of center-focus type and has a center or a focus of order greater than 3 .

It follows from this theorem that for system (3.1) one cannot construct more than 3 small-amplitude limit cycles near a focus by perturbation. 


\section{Bifurcation and Limit Cycles for Kolmogorov Prey-predator and Lotka-Volterra Systems}

\subsection{Cubic Kolmogorov Prey-predator System}

To illustrate the applicability of our approach, in this subsection we discuss briefly the analysis of another two-dimensional biological system, the cubic Kolmogorov prey-predator system constructed and analyzed by Lu and He in [12] using similar algebraic methods. The system is of the form

$$
\begin{aligned}
& \frac{\mathrm{d} x}{\mathrm{~d} t}=x\left(-2-a_{0}+a_{1}+a_{0} x-2 a_{1} x+y+a_{1} x^{2}+x y\right), \\
& \frac{\mathrm{d} y}{\mathrm{~d} t}=y\left(2+a_{2}-x-y-2 a_{2} y+a_{2} y^{2}\right) .
\end{aligned}
$$

This system has a positive steady state $(\bar{x}, \bar{y})=(1,1)$ and it becomes of centerfocus type when $a_{0}=0$. Under the condition $a_{0}=0$, system (4.1) may be transformed by a simple linear transformation $x=Y+X+1, y=-X+1$ into the following canonical form

$$
\begin{aligned}
\frac{\mathrm{d} X}{\mathrm{~d} t}= & Y-a_{2} X^{2}-X Y+a_{2} X^{3} \\
\frac{\mathrm{d} Y}{\mathrm{~d} t}= & -X-\left(2-a_{1}-a_{2}\right) X^{2}+2 a_{1} X Y+\left(1+a_{1}\right) Y^{2}-\left(1-a_{1}+a_{2}\right) X^{3} \\
& -\left(2-3 a_{1}\right) X^{2} Y-\left(1-3 a_{1}\right) X Y^{2}+a_{1} Y^{3} .
\end{aligned}
$$

By computing the Liapunov constants $v_{3}, v_{5}, v_{7}$ of (4.2) and isolating the real zeros of $\left\{v_{3}, v_{5}\right\}$, we can determine $\left(a_{1}, a_{2}\right) \approx(0.08020305719,0.2955574645)$ such that $v_{3}=v_{5}=0$ and $v_{7}<0$; in this case the steady state $(1,1)$ is an asymptotically stable focus of order 3 .

We can perturb (4.2) by adding $\lambda X$ to the first equation and $\lambda Y$ to the second equation, and substituting $a_{1}$ with $a_{1}+\omega$ and $a_{2}$ with $a_{2}+\xi$. Then, compute the Lipunov constants $v_{3}, v_{5}, v_{7}$ of the perturbed system and use DV to derive conditions on $\xi$ and $\omega$ such that $v_{3}<0, v_{5}>0, v_{7}<0$ as we have done in Section 3.2. In this way, we find that, if the perturbation variables take sufficiently small values such that

$$
-0.001<\xi \ll \omega \ll-\lambda<0,
$$

then the Liapunov constants of the perturbed system have alternate signs, i.e.,

$$
v_{1}=\lambda>0, \quad v_{3}<0, \quad v_{5}>0, \quad v_{7}<0 .
$$

In this case, the stability of the focus $(1,1)$ turns over three times and thus three limit cycles may bifurcate in a small neighborhood of $(1,1)$. This conclusion confirms the result given in [12].

\subsection{Bifurcation and Limit Cycles for High-dimensional Systems}

Many biological systems are high-dimensional (e.g., of the form (1.1) with $n>$ 2 ). The analysis of bifurcation and limit cycles for such systems is much more difficult. Some theorems and methods such as the generalized Poincaré-Bendixon 
theorem and the describing function method may be applied for the analysis (see, e.g., [17]), but they are applicable only to certain high-dimensional systems. The methods of center manifold $[4,10]$ and Liapunov-Schmidt reduction [5] allow one to reduce any system of dimension $n>2$ to a planar system without losing any significant aspect of the dynamic characters. These methods in combination with the method for planar systems presented in Section 2 can be used for the algebraic analysis of bifurcation and limit cycles for high-dimensional systems. Here we use the method of center manifold, which is convenient for our treatment of planar systems using Liapunov constants.

To perform reduction, we need the condition under which the Jacobian matrix $\boldsymbol{J}$ of system (1.1) has a pair of purely imaginary eigenvalues and all the other eigenvalues with negative real parts. Such a condition may be given in terms of the Hurwitz determinants and the constant term of the characteristic polynomial of $\boldsymbol{J}$ according to a simple criterion established by El Kahoui and Weber [7]. The criterion was derived for an arbitrary univariate polynomial $A$ to have one pair of purely imaginary roots and all the other roots with negative real parts by linking the Hurwitz determinants $\Delta_{i}$ of $A$ to the principal subresultant coefficients of $A_{2}$ and $A_{1}$, where $A_{1}\left(\lambda^{2}\right)+\lambda A_{2}\left(\lambda^{2}\right)=A(\lambda)$, and by investigating the behavior of $\Delta_{i}$ in the case where $A$ has symmetric roots. Under the condition determined by using El Kahoui and Weber's criterion [7, Theorem 3.6], we can transform system (1.1) to a system of special form and then reduce the transformed system to a two-dimensional system of center-focus type by using the center manifold theorem. We will see how the reduction proceeds from the example in the following subsection. Bifurcation and limit cycles for the obtained two-dimensional system may be analyzed by using the method explained in Section 2.

\subsection{Competitive Three-dimensional Lotka-Volterra System}

In this subsection, we use a competitive three-dimensional Lotka-Volterra system (which models three mutually competing species) to illustrate the analysis of bifurcation and limit cycles by using the center manifold theorem and algebraic methods. The system has the form

$$
\begin{aligned}
& \frac{\mathrm{d} x_{1}}{\mathrm{~d} t}=x_{1}\left[\left(1-x_{1}\right)+\left(1-x_{2}\right)+\left(1-x_{3}\right)\right], \\
& \frac{\mathrm{d} x_{2}}{\mathrm{~d} t}=x_{2}\left[\left(1-x_{1}\right)+\left(1-x_{2}\right)+2\left(1-x_{3}\right)\right], \\
& \frac{\mathrm{d} x_{3}}{\mathrm{~d} t}=x_{3}\left[\mu_{1}\left(1-x_{1}\right)+\mu_{2}\left(1-x_{2}\right)+3\left(1-x_{3}\right)\right],
\end{aligned}
$$

where $\mu_{1}$ and $\mu_{2}$ are two real parameters. This system has been studied in [26, 13] (see also [17]) and it has a unique positive steady state $(1,1,1)$.

By using the transformation $x=x_{1}-1, y=x_{2}-1, z=x_{3}-1$ and rewriting the transformed system of (4.3) in the vector form, we obtain

$$
\frac{\mathrm{d} \boldsymbol{x}}{\mathrm{d} t}=\boldsymbol{D} \boldsymbol{A} \boldsymbol{x},
$$


where

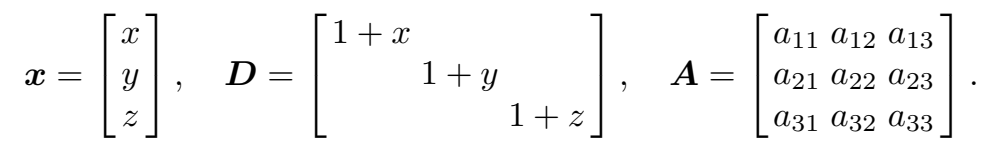

The Jacobian matrix of $(4.3)$ at $(1,1,1)$ is the same as $\boldsymbol{A}$. It is not difficult to prove that the characteristic polynomial of $\boldsymbol{A}$ has a negative real root and a pair of purely imaginary roots if and only if

$$
\operatorname{det}(\boldsymbol{A})=\left(\boldsymbol{A}_{11}+\boldsymbol{A}_{22}+\boldsymbol{A}_{33}\right) \operatorname{tr}(\boldsymbol{A})<0,
$$

where

$$
\begin{array}{ll}
\operatorname{tr}(\boldsymbol{A})=a_{11}+a_{22}+a_{33}, & \boldsymbol{A}_{11}=a_{22} a_{33}-a_{23} a_{32}, \\
\boldsymbol{A}_{22}=a_{11} a_{33}-a_{13} a_{31}, & \boldsymbol{A}_{33}=a_{22} a_{11}-a_{12} a_{21} .
\end{array}
$$

Condition (4.5) is equivalant to

$$
10-3 \mu_{2}-2 \mu_{1}=0, \quad \mu_{2}-\mu_{1}<0 .
$$

Under this condition and substituting $5-\frac{3}{2} \mu_{2}$ for $\mu_{1}$, we may construct a transformation matrix $\boldsymbol{T}$ according to the method described in [13], and system (4.3) may be transformed by the transformation $\boldsymbol{T} \boldsymbol{x} \rightarrow \boldsymbol{x}$ into the following form

$$
\begin{aligned}
& \frac{\mathrm{d} x}{\mathrm{~d} t}=-\frac{3\left(\mu_{2}-2\right)}{2\left(\mu_{2}-4\right)} x-\frac{2 \mu_{2}-5}{\mu_{2}-4} y+l_{1}\left(x, y, z, \mu_{2}\right), \\
& \frac{\mathrm{d} y}{\mathrm{~d} t}=-\frac{\left(\mu_{2}-10\right)\left(\mu_{2}-2\right)}{4\left(\mu_{2}-4\right)} x+\frac{3\left(\mu_{2}-2\right)}{2\left(\mu_{2}-4\right)} y+l_{2}\left(x, y, z, \mu_{2}\right), \\
& \frac{\mathrm{d} z}{\mathrm{~d} t}=-5 z+l_{3}\left(x, y, z, \mu_{2}\right),
\end{aligned}
$$

where $l_{i}\left(x, y, z, \mu_{2}\right)$ are the terms nonlinear in $x, y, z$. Then by the center manifold theorem [10, p. 152], we may analyze the bifurcation and limit cycles for system (4.6) on a two-dimensional center manifold. The center manifold up to approximation of order $k$ can be described as

$$
z=\Psi(x, y)=\sum_{i=2}^{k} \sum_{j=0}^{i} c_{i j} x^{i-j} y^{j}+\text { h.o.t. }
$$

where h.o.t. denotes the terms of higher order $(>k)$. It follows that

$$
\frac{\mathrm{d} z}{\mathrm{~d} t}=\frac{\partial \Psi}{\partial x} \frac{\mathrm{d} x}{\mathrm{~d} t}+\frac{\partial \Psi}{\partial y} \frac{\mathrm{d} y}{\mathrm{~d} t} .
$$

To compute for instance the first two Liapunov constants of the two-dimensional system (to be determined), we need to consider the center manifold up to quartic approximation. Substituting

$$
\frac{\mathrm{d} z}{\mathrm{~d} t}=-5 z+l_{3}\left(x, y, z, \mu_{2}\right) \text { with } z=\sum_{i=2}^{4} \sum_{j=0}^{i} c_{i j} x^{i-j} y^{j}
$$

into (4.8) and comparing the coefficients of the two sides of the two equations in $x, y, \mathrm{~d} x / \mathrm{d} t, \mathrm{~d} y / \mathrm{d} t$, we obtain a system of linear equations in $c_{i j}(i=2, \ldots, 4, j=$ 
$0, \ldots, 2)$. Then $c_{i j}$ may be found as rational functions $\bar{c}_{i j}$ of $\mu_{2}$, which are quite complicated (for example, the numerator and the denominator of $\bar{c}_{20}$ both consist of 29 terms and are of degree 28 in $\left.\mu_{2}\right)$. Substituting $z=\left.\Psi(x, y)\right|_{c_{i j}=\bar{c}_{i j}}$ into the first two equations in (4.6) results in a two-dimensional system of center-focus type. This system may be further transformed to the canonical form by the linear transformation

$$
x=\frac{4\left(\mu_{2}-4\right)}{\left(\mu_{2}-10\right)\left(\mu_{2}-2\right)} Y-\frac{6}{\left(\mu_{2}-10\right) \delta} X, \quad y=-\frac{1}{\delta} X, \quad t=\frac{\tau}{\delta},
$$

where $\delta=\sqrt{-\mu_{2} / 2+1}$. Then by using the Epsilon function miscel[licon], we can compute the first two Liapunov constants $v_{3}$ and $v_{5}$ of the two-dimensional system in the canonical form. ${ }^{3}$ The numerators $\bar{v}_{3}, \bar{v}_{5}$ of $v_{3}, v_{5}$ and the denominator of $v_{5}$ consist of 44,197 and 35 terms and are of total degrees 16,39 and 45 in $\delta, \mu_{2}$, respectively. Computation using RS shows that $\left\{\bar{v}_{3}, \delta^{2}+\mu_{2} / 2-1\right\}$ has 7 isolated real zeros for $\left(\mu_{2}, \delta\right)$. Let the physical parameters $\mu_{2}$ and $\mu_{1}=5-\frac{3}{2} \mu_{2}$ be assumed positive. Then there are two real values $\bar{\mu}_{2}^{\prime}$ and $\bar{\mu}_{2}^{\prime \prime}$ for $\mu_{2}$ such that $\operatorname{det}(\boldsymbol{A})<0$, where

$$
\bar{\mu}_{2}^{\prime} \in\left[\frac{12946800109}{8589934592}, \frac{25893600219}{17179869184}\right]=I, \quad \bar{\mu}_{2}^{\prime \prime}=\frac{8}{5} .
$$

It is easy to verify by using RS that $\bar{v}_{3}=0, v_{5}>0$ at $\bar{\mu}_{2}^{\prime}, v_{3}<0$ at the right end of $I$, and the denominator of $v_{3}$ has no real root in $I$. Therefore, the steady state $(1,1,1)$ is unstable and one can perturb $\bar{\mu}_{2}^{\prime}$ slightly to $\bar{\mu}_{2}^{*}$ for $\mu_{2}$ such that $v_{3}<0$, while $v_{5}>0$ remains true, so that one limit cycle bifurcates. Next, perturb $5-\frac{3}{2} \bar{\mu}_{2}^{*}$ slightly to $\bar{\mu}_{1}^{*}$ for $\mu_{1}$ such that $v_{3}<0, v_{5}>0$ remain true, while the real part of the two conjugate complex eigenvalues of the Jacobian matrix of the two-dimensional system becomes positive. Then the second limit cycle bifurcates. Our construction of two limit cycles here is similar to the constructions in [13].

For the other value $\bar{\mu}_{2}^{\prime \prime}=8 / 5$ of $\mu_{2}, \operatorname{det}(\boldsymbol{A})<0$ and $v_{3}=v_{5}=0$, so the steady state is likely to be a center (see $[26]$ ).

Analysis of bifurcation and limit cycles for high-dimensional systems by using the method of center manifold involves complicated calculations and the produced planar systems are usually large, so the Liapunov constants become very large and unmanageable. For example, to construct three small-amplitude limit cycles for a three-dimensional Lotka-Volterra system one needs to compute the first three Liapunov constants and thus the center manifold needs to be approximated up to order 6 . In this case, the computation of the Liapunov constants of the produced planar system even becomes rather difficult.

\footnotetext{
${ }^{3}$ Most of the computations discussed in this paper were performed step by step on a laptop T2400 with 2 CPUs, $1.83 \mathrm{GHz}$ and $987 \mathrm{MHz}$, and $2 \mathrm{~GB}$ RAM. Some of them were verified by using different software tools. Each successful step of computation usually takes no more than a few minutes. However, the computation of $v_{5}$ here requires nearly one hour.
} 


\section{Concluding Remarks}

In this paper, we have shown how to analyze bifurcation and limit cycles for two biological systems of dimension 2 and another of dimension 3 using an algebraic approach based on the methods of triangular decomposition, Gröbner bases, discriminant varieties, real solution classification, and quantifier elimination by partial CAD. Several efficient software packages including Epsilon, FGb, RS, DV, and DISCOVERER in which some of these algebraic methods are implemented have been used for the involved symbolic computations with semi-algebraic systems. The analysis of bifurcation and limit cycles is presented in detail for the cubic self-assembling micelle system with chemical sinks and briefly for the cubic Kolmogorov prey-predator system, both two-dimensional. It is proved that the self-assembling micelle system may have a focus of order 3 , from which three limit cycles can be constructed by small perturbation, but this system cannot have a focus of order greater than 3. Bifurcation analysis for the competitive LotkaVolterra system is carried out by using the method of center manifold that reduces the three-dimensional system to a two-dimensional one. Two limit cycles are then constructed from the determined focus of order 2 by small perturbation.

The investigations described in this paper illustrate the applicability and limitation of our algebraic approach for the analysis of bifurcation and limit cycles for biological systems. This approach uses exact symbolic computation and ensures that all the obtained results are mathematically rigorous. It is a useful alternative to the experimental approach based on numerical simulation and visualization.

Although the algebraic methods underlying our approach have been well developed and are powerful, it is well known that symbolic computations involved in these methods are very heavy in general. How to improve the methods by introducing new and specialized algebraic techniques, how to use them to analyze bifurcation and limit cycles for high-dimensional biological systems more effectively, and how to deal with biological systems of the form (1.1) with $p_{i}$ being rational or other functions are some of the questions that remain for future research.

\section{References}

1. Andronov, A. A., Leontovich, E. A., Gordon, I. I., Maier, A. G.: Theory of Bifurcations of Dynamic Systems on a Plane. Israel Program of Scientific Translations, Jerusalem (1971).

2. Ball, R., Haymet, A. D. J.: Bistability and hysteresis in self-assembling micelle systems: Phenomenology and deterministic dynamics. Phys. Chem. Chem. Phys. 3: 4753-4761 (2001).

3. Buchberger, B.: Gröbner bases: An algorithmic method in polynomial ideal theory. In: Multidimensional Systems Theory (N.K. Bose, ed.), pp. 184-232. Reidel, Dordrecht (1985).

4. Carr, J.: Applications of Center Manifold Theory. Springer, New York (1981).

5. Chow, S.-N., Hale, J.K.: Methods of Bifurcation Theory. Springer, New York (1982). 
6. Collins, G. E., Hong, H.: Partial cylindrical algebraic decomposition for quantifier elimination. J. Symb. Comput. 12: 299-328 (1991).

7. El Kahoui, M., Weber, A.: Deciding Hopf bifurcations by quantifier elimination in a software-component architecture. J. Symb. Comput. 30: 161-179 (2000).

8. Farr, W. W., Li, C., Labouriau, I. S., Langford, W. F.: Degenerate Hopf bifurcation formulas and Hilbert's 16th problem. J. Math. Anal. 20(1): 13-30 (1989).

9. Faugère, J.-C.: A new efficient algorithm for computing Gröbner bases $\left(F_{4}\right)$. $J$. Pure Appl. Algebra 139: 61-88 (1999).

10. Kuznetsov, Y.A.: Elements of Applied Bifurcation Theory (2nd edn.). Springer, New York (1998).

11. Lazard, D., Rouillier, F.: Solving parametric polynomial systems. J. Symb. Comput. 42: 636-667 (2007).

12. Lu, Z., He, B.: Multiple stable limit cycles for a cubic Kolmogorov prey-predator system. J. Eng. Math. 18(4): 115-117 (2001).

13. Lu, Z., Luo, Y.: Two limit cycles in three-dimensional Lotka-Volterra systems. Comput. Math. Appl. 44(1/2): 51-66 (2002).

14. Miller, R. K., Michel, A. N.: Ordinary Differential Equations. Academic Press, New York London (1982).

15. Niu, W.: Application of quantifier elimination and discriminant varieties to stability analysis of biological systems. In: Proceedings MACIS 2006 (D. Wang and Z. Zheng, eds.), pp. 243-253. Beihang University, China (2006).

16. Niu, W., Wang, D.: Algebraic approaches to stability analysis of biological systems. Math. Comput. Sci. 1(3): 507-539 (2008).

17. Shahruz, S. M., Kalkin, D. A.: Limit cycle behavior in three- or higher-dimensional non-linear systems: The Lotka-Volterra example. J. Sound Vibration 246(2): 379386 (2001).

18. Shi, S.: A concrete example of the existence of four limit cycles for plane quadratic systems. Sci. Sinica (A) 23: 153-158 (1980).

19. Wang, D.: A class of cubic differential systems with 6-tuple focus. J. Diff. Eqns. 87: 305-315 (1990)

20. Wang, D.: Mechanical manipulation for a class of differential systems. J. Symb. Comput. 12: 233-254 (1991).

21. Wang, D.: Elimination Methods. Springer, Wien New York (2001).

22. Wang, D.: Elimination Practice: Software Tools and Applications. Imperial College Press, London (2004).

23. Wang, D., Xia, B.: Stability analysis of biological systems with real solution classification. In: Proceedings ISSAC 2005 (M. Kauers, ed.), pp. 354-361. ACM Press, New York (2005).

24. Wang, D., Xia, B.: Algebraic analysis of stability for some biological systems. In: Proceedings AB 2005 (H. Anai and K. Horimoto, eds.), pp. 75-83. Universal Academy Press, Tokyo (2005).

25. Wu, W.-t: Mathematics Mechanization. Science Press/Kluwer, Beijing (2000).

26. Xiao, D., Li, W.: Limit cycles for the competitive three dimensional Lotka-Volterra system. J. Diff. Eqns. 164: 1-15 (2000).

27. Yang, L., Xia, B.: Real solution classifications of parametric semi-algebraic systems. In: Algorithmic Algebra and Logic - Proceedings A3L 2005 (A. Dolzmann, A. Seidl, and T. Sturm, eds.), pp. 281-289. Herstellung und Verlag, Norderstedt (2005).

28. Ye, Y., Cai, S., Chen, L., Huang, K., Luo, D., Ma, Z., Wang, E., Wang, M., Yang, X.: Theory of Limit Cycles. Amer. Math. Soc., Providence, RI (1986).

29. Zhang, Z., Ding, T., Huang, W., Dong, Z.: Qualitative Theory of Differential Equations. Amer. Math. Soc., Providence, RI (1992). 